\title{
Poétique de l'hybride dans la littérature postexilique de Kim Thúy
}

\author{
Julien Defraeye \\ Université de Waterloo
}

À la suite de l'essoufflement de la question nationale dans les années 1980, la littérature québécoise a témoigné de la montée des écriture migrantes, ainsi que les nomma le poète Robert Berrouët-Oriol en 1986. Notons des écrits comme La Québécoite (1983) de Régine Robin, Comment faire l'amour avec un Nègre sans se fatiguer (1985) de Dany Laferrière, La Mémoire de l'eau (1992) de Ying Chen et Le Pavillon des miroirs (1995) de Sergio Kokis, qui suggèrent tous un renouvellement esthétique littéraire faisant rupture avec la tradition, en insufflant de nouveaux enjeux : flux migratoires, exils, débats sur l'identité et 
l'altérité au Québec, pour ne citer que les plus prégnants. Robert Dion, en 1997, note que «la littérature québécoise se laisse [alors] imprégner par les mouvements mondiaux, particulièrement par la pensée de la postmodernité - une pensée déterritorialisée et déshistoricisée, propice à toutes les hybridations » (p. 189). Même si une certaine saturation de la catégorie se fait ressentir à partir des années 2000, certains écrivains entrent encore en résonance avec ses problématiques littéraires qui ont marqué la fin du siècle dernier. Kim Thúy, à travers sa plume d'origine vietnamienne, reflète la persistance de ces questions dans l'écrit québécois, même si celle-ci reste toute relative. ru (2009), mãn (2013) et vi (2016) expriment ainsi tous trois ce décloisonnement des problématiques en contexte post-national et leur propension à une influence culturelle réciproque. Dans ces romans que l'on qualifiera maintenant de postexiliques ${ }^{1}$ pour "décrire l'aspect singulier de récits qui ne sont plus entravés par les formes traditionnelles du déplacement » (Harel, 2005, p.20), Kim Thúy propose une nouvelle poétique de l'hybride dans un Québec où l'autre a toujours été multiple. Dans cet article, je me pencherai sur l'importance de la trace évoquée par Pierre Nepveu dans les écritures migrantes, qui se manifeste principalement chez Kim Thúy dans les domaines linguistique et culinaire. À travers la persistance des débats identitaires dans notre corpus, je reviendrai également sur le rapport à l'altérité québécoise avant de me focaliser sur l'hybridation culturelle dans les romans de Kim Thúy.

\footnotetext{
1 Simon Harel (2005) évoque l'évolution des dénominations usitées pour désigner ce type d'écrit, de "littérature des communautés culturelles»au « postexilique », en passant par les « écritures migrantes » de Berrouët-Oriol.
} 


\section{L'importance de la trace linguistique}

Pierre Nepveu remarquait en 1988, dans L'Écologie du réel, que l'imaginaire migrant au Québec s'offre comme « un univers de traces, de marques concrètes» (p. 202). De la pluralité de l'identité migrante sourdent des témoins très matériels du déchirement dans sa littérature. Leur expression se joue alors « dans des circonstances particulières, et à travers des tensions, des paradoxes, des limites » (p. 202) Bien que très différents dans leurs narrations, les romans de Kim Thúy sont le constat d'une césure géographique première, celle de la diaspora vietnamienne au Québec. ru s'empare de l'histoire - que l'on pourrait presque croire autobiographique - de Nguyễn An Tịnh, une Vietnamienne de Granby qui pose rétrospectivement son regard sur son histoire personnelle, qui s'inscrit inévitablement dans l'histoire collective de la fuite des boat people dans les années 1970. mãn se distancie légèrement de cette expérience traumatique en narrant la relation sentimentale, mais aussi le processus hybride d'acculturation d'une Vietnamienne arrivée à l'âge adulte au Québec après une enfance sur les rives du Mékong. vi, son roman éponyme le plus récent, raconte le passage à la vie d'adulte d'une adolescente exilée timide, entourée de sa mère et ses frères, entre Québec et Montréal. Ce tiraillement des personnages de Kim Thúy, qui est avant tout spatial, est emblématique de l'écriture migrante : Régine Robin, d'origine française, stipulait par exemple dans L'Amour $d u$ yiddish qu'elle habiterait toujours un "entre-deux»: «Incurable, je n'écris que d'un lieu, celui de l'“entre”, l'entredeux, l'entre-deux-océans, l'entre-deux-langues, l'entre-deuxidéologies » (p. 29). 
L'entre-deux-langues, système hybride et pluriel du langage, est une trace majeure du déchirement topique chez Kim Thúy. La langue maternelle, ici le vietnamien, se perd progressivement, et la réinscription dans un système langagier autre ne se fait que de manière partielle. Les personnages de ses romans restent irrémédiablement au stade d'apprenants du français au Québec tout en polarisant parfois avec regrets leurs rapports au vietnamien: "J'ai dû réapprendre ma langue maternelle, que j'avais abandonnée trop tôt.» (ru, p.88) L'hybridité du système linguistique se matérialise dans une traduction quasi constante d'expressions de la langue source, souvent en marge du texte. $r u$ s'ouvre sur une première occurrence de ce phénomène, à l'appui du dictionnaire : «En français, ru signifie "petit ruisseau" et, au figuré "écoulement (de larmes, de sang, d'argent)" »(Le Robert historique). En vietnamien, ru signifie "berceuse", "bercer".» (ru, p. 7) mãn reprend également cette tension du langage, cette fois dans la marge physique de la page. Certains mots de vocabulaire sont traduits autour du texte, même quand ceux-ci ne sont pas mentionnés en son sein, pour témoigner d'une contextualisation authentique in situ. Certains éléments culturels spécifiques au pays d'origine sont donnés dans leur langue d'appartenance, accompagnés d'une équivalence dans la langue d'accueil, comme le áo dài (mãn, p. 20), tunique échancrée vietnamienne, que la mère de la narratrice de mãn porte avec élégance. D'autres pratiques culturelles sont également présentées parallèlement dans les deux langues, comme ce poème traditionnel connu de tous les vietnamiens, répété inlassablement par sa mère :

Trong đầm gì đẹp bằng sen

Lá xanh, bông trắng lại chen nhụy vàng, 
Nhụy vàng, bông trắng, lá xanh,

Gần bùn mà chẳng hôi tanh mùi bùn.

Dans le marais, quoi de plus beau que le lotus,

Où rivalisent feuilles vertes, pétales blancs et pistils jaunes,

Pistils jaunes, pétales blancs, feuilles vertes,

Près de la boue, mais sans sa puanteur. (mãn, p. 81)

Quelques pages plus loin, ce poème de Roumi, célèbre poète persan du XIII e siècle, surgit au détour d'une conversation :

A fine hanging apple

in love with your stone,

the perfect throw that clips my stem.

Une jolie pomme suspendue

en amour avec votre galet,

le lancer parfait qui coupa mon pédoncule. (mãn, p. 87)

Cependant, le processus de traduction prend quelques détours et fait parfois face à des situations qui se présentent comme insolubles. La polysémie de certains vocables crée ainsi des zones troubles qui mènent à un questionnement que la narratrice de mãn reprend bien volontiers. Le vietnamien $M e$ Ghẻ peut par exemple se traduire comme "mère froide " ou comme «mère galeuse » (mãn, p. 23), influant de ce fait plus ou moins directement sur le sens et l'idée que le lecteur se fait de sa belle-mère. Visiblement volontaire dans son apprentissage de la langue seconde, la narratrice s'intéresse tout particulièrement au cheminement étymologique des mots, jusqu'au moment où ceux-ci laissent entrevoir certains illogismes sous un regard neuf :

Je devine, je tâte, et la réponse est aussi souvent la bonne que la mauvaise. Je fais constamment des erreurs, et jusqu'à ce jour, la plus étonnante a porté sur le mot « rebelle », que je croyais être un dérivé de "belle»: être belle de nouveau parce que la beauté s'acquiert et se perd. (mãn, p. 92) 
La transition d'un système à l'autre bute sur les particularités langagières où les incohérences d'un apprentissage trop formaté : «Entre le français et l'anglais, les faux-amis tendent leurs pièges et, chaque fois, je succombais. » (mãn, p. 123) Le processus de traduction lutte alors entre deux fonctionnements linguistiques hétéroclites et dont les équivalences ne seront que nécessairement approximatives. La narratrice de vi rappelle que "même si le vietnamien s'écrit aujourd'hui au son, la plupart des mots portent encore la trace des images originelles des idéogrammes» (vi, p. 30). Elle s'intéresse par ailleurs à décomposer les idéogrammes chinois quelques pages plus loin - pour rejoindre son mari en poste à Shanghai - en analysant leurs dérivés et associations :

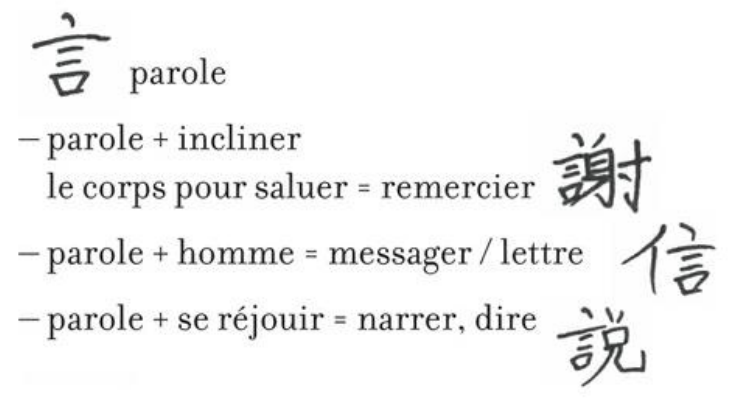

(vi, p. 70)

La distance émerge également à travers la richesse lexicale qui ne se retrouve pas nécessairement d'une langue à l'autre. La narratrice de ru estime que «le geste d'aimer doit être traduit d'une langue à l'autre, il doit être appris » (ru, p. 104), constante reprise dans vi, qui s'attarde sur la pluralité lexicale du verbe «adorer » en vietnamien : « adorer à la folie, adorer au point de figer comme un arbre, adorer jusqu'à en perdre connaissance, jusqu'à la fatigue, jusqu'à l'abandon de 
soi » (vi, p. 104). Les situations cocasses - mais positives - se multiplient, notamment quand l'apprentissage voit ses limites quant à la pratique effective de la langue: l'idiomatique, par exemple, amène à des incompréhensions ponctuelles: «La première fois que j'ai entendu au Québec l'expression "tu es dans ma bulle", je pensais que mon interlocuteur me déclarait son amitié en me permettant d'être dans ses pensées, dans son espace intérieur, alors qu'il voulait en fait que je m’en dégage. » (vi, p. 59) Au Vietnam, la proximité phonétique de certains vocables et l'importance de la différenciation par les accents engendrent ici et là des dissonances qui pointent dans la narration parmi les touristes qui se rendent en Asie, comme pour les mots «uriner» et «poivre»: «Ces deux mots ne se différencient que par un accent, un ton presque imperceptible aux oreilles non entraînées. Un simple accent pour un simple moment de bonheur. » (ru, p. 129) Malgré les dispositions où la traduction se révèle ambiguë, voire truculente par moments, ce processus demeure tantôt inerte, puisque tout bonnement impossible. La langue d'origine reflète une réalité premièrement culturelle, qui s'inscrit dans un contexte spatial ou géographique, mais, dans vi, qui se consigne également dans une expérience du temps - le temps historique - dont elle a bien du mal à se défaire: «La langue vietnamienne que je connaissais était marquée par l'exil et figée dans une ancienne réalité, celle d'avant la présence des Soviétiques et des liens étroits avec Cuba, la Bulgarie, la Tchécoslovaquie, la Roumanie... » (vi, p. 100-101) La narratrice de $v i$ est de ce fait le témoin vivant d'un langage et d'une histoire figés et morts dans le temps au moment de son départ pour le pays d'accueil. À l'opposé, dans le procès linguistique et cognitif qui caractérise la découverte de la langue du pays hôte, Kim Thúy associe le langage au vivant à travers des 
figures récurrentes dans ru et mãn, qui s'apparenteraient à des allégories du langage. Ces adjuvants, souvent plus âgés, détiennent le langage et sont la clé de la source de transmission du français pour ces narratrices migrantes. Dans $r u$, il s'agit de monsieur Minh, propriétaire d'un restaurant chinois de la rue Côte-des-Neiges, où le père de la narratrice travaillait comme livreur, qui lui a «transmis le désir d'écrire » (ru, p. 97) :

Lui, ce n'était pas le ciel qui l'avait sauvé, c'était l'écriture. Il avait écrit plusieurs livres pendant ses années au camp de rééducation, et ce, toujours sur le seul et unique bout de papier qu'il possédait, une page par-dessus l'autre, un chapitre après l'autre, une histoire sans suite [...] il me récitait les mots de son dictionnaire personnel : nummulaire, geindre, quadriphonie, in extremis, sacculine, logarithmique, hémorragie..., comme un mantra, comme une marche vers le vide. ( $r u$, p. 97)

La narratrice de mãn raconte également avoir pour voisin celui qu'elle nomme « un dictionnaire vivant» (mãn, p. 59) :

Les voisins le considéraient comme fou parce qu'il se plaçait tous les jours sous le jambosier, où il récitait des mots en français et leurs définitions. Son dictionnaire avait été tenu contre lui pendant toute sa jeunesse, avait été confisqué mais il continuait à tourner les pages dans sa tête [...].

- Humer : aspirer par le nez pour sentir. Humer l'air. Humer le vent. Humer le brouillard. Hume le fruit ! Hume ! Jambose, aussi appelée pomme d'amour en Guyane. Hume! (mãn, p. 59)

La narratrice se trouve alors happée par la langue, qui persiste comme trace bien après la transition dans le pays d'accueil. 


\section{Médiations culinaires}

Une autre marque majeure vient sourdre dans la poétique de l'hybride de Kim Thúy à travers l'univers culinaire qui l'habite. Ceux qui se sont penchés sur le parcours personnel de l'auteure québécoise « aux lunettes roses $»^{2}$ se rappelleront ses débuts de chef dans son restaurant $R u$ de Nam sur la rue Notre-Dame de Montréal, bien avant le succès médiatique de son premier roman $r u$. Dans son écriture, les plats traditionnels, qu'ils soient vietnamiens ou non, se présentent comme une première médiation culturelle entre peuples, particulièrement présente dans vi. La cuisine s'érige alors dans le partage de la tradition pour les communautés d'expatriés de Montréal qui célèbrent le Nouvel An avec l'Association des femmes vietnamiennes du Québec au Complexe Desjardins en vendant les « rouleaux, les raviolis farcis, les pâtés chauds, le gâteau au manioc » (vi, p. 81) dans des kiosques. La nourriture est également l'occasion d'une transmission intergénérationnelle des pratiques à travers la confection des plats traditionnels : "Mais la nourriture nous a soudées, sa mère et moi, puisque c'est moi qui ai trouvé par terre la dent qu'elle s'était cassée en s'acharnant sur un ligament des pattes de poulet vendues à la fenêtre de la maison d'en face. » (vi, p. 73) La nourriture prend ici la dimension d'un héritage culturel primordial, vecteur d'une tradition qui se transmet de génération en génération. De plus, Kim Thúy confère spontanément une visée historique à la découverte de la nourriture. Le domaine du culinaire est ainsi le terrain d'un réinvestissement de l'histoire collective à travers le partage de la nourriture. Les plats s'inscrivent alors dans l'espace comme

\footnotetext{
${ }^{2}$ Comme le remarque Christian Desmeules dans son entretien avec l'auteure pour Le Devoir en mars 2013.
} 
dans le temps par leurs spécificités, et leur découverte permet à la narratrice de revisiter l'histoire de son pays natal, depuis qu'elle l'a quitté :

J'ai tenté de rattraper quelques bribes des vingt ans du Vietnam derrière le rideau de fer en traînant autour des tablesrestaurants. Devant mon hôtel, il y en avait plusieurs. L'une offrait des baguettes au pâté de foie, l'autre, des vermicelles sautés, et plusieurs, des soupes tonkinoises. Je finissais mes journées avec cette soupe qui ne ressemblait en rien à celle cuisinée à Montréal, Los Angeles, Paris, Sydney ou Saigon. La version hanoïenne se vendait seulement avec quelques tranches de bœuf saignant alors que j'avais toujours mangé ce plat avec une dizaine d'ingrédients, dont les tendons, l'estomac, le jarret, du basilic thaï, des fèves germées... (vi, p. 102)

Plus qu'un héritage culturel, le culinaire prend la forme du devoir dans $r u$, dans lequel la narratrice entend défendre la mémoire des hommes enfermés dans les camps de rééducation du régime dans les années 1970 et de leurs femmes, qui leur apportaient ces quelques mets sans savoir s'ils étaient toujours vivants : «En souvenir de ces femmes, je prépare de temps à autre cette viande rissolée pour mes fils, afin de préserver, de répéter, ces gestes d'amour.» (ru, p. 44) Cette tradition fait rupture avec l'alimentation en contexte nord-américain, qui ne marque que très rarement une transmission culturelle ou un devoir de mémoire. La narratrice de vi s'en étonne: "elle ne pouvait que s'empêcher de constater avec tristesse que sa femme américaine le nourrissait surtout de plats surgelés » (vi, p. 124). Les plats surgelés, sériés à l'infini, sont alors le symbole d'une perte identitaire célébrée dans la culture de masse du nouveau continent. Ce refus de l'altérité identitaire dans les plats traditionnels étrangers mène ainsi certains des protagonistes à mentir aux frontières en ne déclarant pas la nourriture qu'ils transportent - «I have no food » (vi, p. 124) -, 
avouant de ce fait une certaine acculturation masquée par le délaissement des pratiques culinaires vietnamiennes. Par un phénomène contigu, le rapport au culinaire témoigne de façon analogue d'une altérité culturelle qui est régulièrement synonyme de voyage et de découverte, au minimum symbolique. Dans vi toujours, les exemples de ces ailleurs culinaires sont pléthores. Le père de la narratrice, diplomate, ramène de ses voyages des spécialités méconnues au Vietnam : «Mon père nous faisait découvrir des délices rapportés d'ailleurs, allant des grains d'anis de Flavigny au foie gras, en passant par les cantaloups parfois disponibles dans certains restaurants français de Saigon. » (vi, p. 42) Cette découverte de l'espace par la nourriture génère une cartographie culinaire de l'espace, le lieu n'offrant parfois de réalité que par sa spécialité alimentaire. Quelques pages plus loin, la nourriture est associée à d'autres pratiques culturelles inscrites dans l'espace, par l'intermédiaire de Tân, un ami des frères de la narratrice :

Tân nous a initiés aux spaghettis alla carbonara, donc à la pancetta et au parmigiano. Il entonnait des chansons en italien et imitait Pavarotti. Il nous a fait découvrir La Dolce Vita et tous les autres films de Marcello Mastroianni. Il a montré à ma mère et à moi le paso doble, le tango, le cha-cha-cha. Black Magic Woman de Santana tourne encore dans sa tête au rythme de ses « un, deux, cha-cha-cha ». (vi, p. 84)

Cela étant, le culinaire se révèle toujours ancré dans l'espace et permet parfois une évasion, aussi fictive qu'elle soit. Le frère de la narratrice est ainsi embauché dans un restaurant japonais où « il [fait] voyager les convives jusqu'à Kobé » (vi, p.51). Malgré ses origines vietnamiennes, «sa manipulation acrobatique des ingrédients lui accord[e] une identité japonaise [...] et les clients nourriss[ent] leurs rêves d'exotisme " (vi, p. 52). Pourtant, le culinaire trace une délimitation claire entre 
le pays d'origine et le pays d'accueil ainsi qu'une marque identitaire qui renouvelle le débat sur l'altérité au Québec : «Il va de soi que certains goûts sont exclusifs et tracent une forte frontière identitaire. » (mãn, p. 80)

\section{Une altérité renouvelée}

L'émergence des écritures migrantes des années 1980 a fragilisé la tradition québécoise, qui s'inscrivait auparavant en littérature périphérique de l'incontestable centre éditorial parisien. Les récits de Robin, de Laferrière ou encore de Kokis ont de ce fait aménagé un espace secondaire dans une littérature québécoise dont les discours n'étaient que rarement contestés, comme le montrent la persistance du terroir jusqu'au milieu des années 1940 et celle de la problématique du projet national. Les écritures migrantes, garantes tangibles de diversité discursive, se sont placées en marge d'une littérature déjà minoritaire et ont alors contribué à renforcer le champ québécois comme littérature majeure : «Ainsi, créant sa propre périphérie, la littérature québécoise aurait solidifié ses assises identitaires. » (Harel, 2005, p. 23) Parallèlement, la littérature québécoise s'est investie des questionnements sur l'identité et l'altérité, rajoutant en complexité dans le débat sur la transculture et le pluralisme qui la caractérise, et surtout la capacité du Québécois à déterminer lui-même s'il est l'autre. Pierre Nepveu évoque ainsi «l'ambiguïté de ce rapport québécois à l'altérité, par le glissement qui va de "reconnaître l'autre" à "être l'autre" » (p. 202). Cette problématique inhérente à la trans-culture est reprise dans les romans de Kim Thúy. Le personnage migrant s'efface alors ponctuellement au 
profit de l'image du migrant, en faisant fi de ces spécificités. Dans mãn, la négation de la pluralité identitaire québécoise relègue certains migrants aux marges : «Il était de ceux qui ont vécu trop longtemps au Vietnam pour devenir canadiens. Et, à l'inverse, qui ont vécu trop longtemps au Canada pour être vietnamiens de nouveau. » (mãn, p. 14) À travers ce refus de l'altérité par l'amalgame de l'identité à l'identitaire et du singulier au collectif, le danger se situe alors dans la remise en question de l'ipséité des écritures migrantes: " N'y a-t-il pas danger, ici, de décrire des processus impersonnels, globaux, qui ne tiennent pas assez compte de ce qui se passe dans les sujets concrets?» (Biron, Dumont et Nardout-Lafarge, 2007, p. 567) Comme une mise en garde à la lecture, Pierre Nepveu rappelle : « Il n'y a pas un texte migrant. » (p. 199)

Une spécificité de l'écriture transculturelle de Kim Thúy semble relever de son attachement pour le nom et la symbolique qu'on lui confère. L'onomastique est évidemment une pratique ancrée dans une culture et une langue, et l'importance du nom varie de l'une à l'autre, comme en témoigne l'exil du Vietnam au Québec pour les protagonistes de Kim Thúy. Dans la traversée des boat people, ru dénonce l'anonymat comme un affront face à la mort, mais surtout une perte identitaire flagrante: «Les autres, qui avaient coulé pendant la traversée, n'avaient pas de noms. Ils sont morts anonymes. » (ru, p.24) puisque le nom est un héritage qui se transmet d'une génération à l'autre : «Il exprimait sa fierté d'avoir créé à la fois un empire et une réputation enviables en répétant son nom pour chacun de ses enfants : «Thérèse Lê Văn An, Jeanne Lê Văn An, Marie Lê Văn An... et mon père, Jean Lê Văn An.» (vi, p. 12) Cependant, le glissement identitaire du migrant infère une dissolution de l'onomastique évidente par le 
passage d'une langue à l'autre : "L'Histoire du Vietnam, avec un grand $\mathrm{H}[\ldots]$ a jeté les accents de nos noms à l'eau [...]. Elle a aussi dépouillé nos noms de leurs sens, les réduisant à la fois à des sons étrangers et étranges dans la langue française. » (ru, p.12) vi révèle pourtant que l'onomastique peut montrer certaines défaillances avant même la transition dans l'espace :

Mon prénom, Bảo Vi, illustrait l'intention de mes parents de «protéger la plus petite». Si l'on traduit littéralement, je suis «Précieuse minuscule microscopique ». Comme dans la plupart des cas au Vietnam, je n'ai pas su être à l'image de mon nom. Souvent, les filles qui s'appellent «Blanche» (Bạch) ou " Neige » (Tuyết) ont le teint très foncé, et les garçons nommés « Puissance » (Hùng) ou « Fort » (Mạnh) craignent les grandes épreuves. Quant à moi, je grandissais sans cesse dépassant de loin la moyenne et, du même élan, me projetant en dehors des normes. (vi, p. 30)

Pour sa mère prénommée Xuân, le même détournement onomastique a lieu : "Très vite, elle a accepté que les garçons ne lui diraient jamais "Tu es mon printemps" même si son prénom, Xuân, voulait dire "printemps" et qu'elle vivait dans un lieu surnommé "la ville au printemps éternel”. » (vi, p. 18) La tradition vietnamienne confère de ce fait une charge symbolique au prénom et inviterait à envisager l'onomastique non pas comme une science, puisqu'elle révèle des défaillances flagrantes dans les faits, mais comme une prédestination figurative du physique ou de la personnalité. Cependant, Kim Thúy insiste nettement sur les incohérences de ces pratiques millénaires, notamment en ce qui concerne le trauma : "Mon prénom ne me prédestinait pas à faire face aux tempêtes en haute mer et encore moins à partager une paillote dans un camp de réfugiés en Malaisie avec une dame âgée qui pleura jour et nuit pendant un mois sans nous expliquer qui étaient les 
quatorze jeunes enfants qui l'accompagnaient. » (vi, p. 45) Le glissement onomastique d'une langue à l'autre fonctionne alors par une logique d'absorption et de transformation, tout comme les emprunts linguistiques d'un système langagier à un autre :

Mon grand-père n'avait jamais protesté lorsque les enseignants lui donnaient un nom français. Par manque de connaissances, par acte de résistance, ses parents ne lui en avaient pas donné. Alors, dans les classes, d'une année à l'autre, d'un professeur à l'autre, il portait un nouveau nom, Henri Lê Văn An, Philippe Lê Văn An, Pascal Lê Văn An... De tous ces noms, il avait conservé Antoine et transformé Lê Văn An en nom de famille (vi, p. 10)

Malgré l'errance onomastique du grand-père de la narratrice, le prénom vietnamien devient alors patronyme français, dont les générations suivantes ont hérité, bien que son origine se trouve dans la volonté de résister à travers la langue.

Parallèlement, l'altérité se joue dans les césures de la filiation. L'exil, souvent traumatique, s'immisce dans les interstices généalogiques, qui sont semblables à la rupture culturelle impliquée par le passage du Vietnam au Québec. La narratrice de mãn évoque l'attitude désorientée de son gardien face aux figures féminines qui l'entourent :

Sa démarche [...] était celle d'un homme incertain, perdu entre deux mondes. Il ne savait plus s'il devait franchir le seuil avant ou après les femmes. Il ne savait plus si sa voix devait être celle de la marieuse ou la sienne. Ses hésitations lorsqu'il s'est adressé à Maman nous ont toutes terrassées. Il l'appelait pêlemêle «grand-sœur » (Chi $)$, «tante » (Cô) et "grande-tante» (Bác). (mãn, p. 15)

Les incohérences à l'égard des traditions du pays d'origine et de celui d'accueil traduisent un malaise face aux pratiques culturelles. mãn est par ailleurs très emblématique de cette malléabilité de la généalogie post-traumatique puisque la 
narratrice révèle dès les premières pages qu'elle ne connait pas son père : «Moi, je n'ai jamais su qui était mon géniteur. » (mãn, p. 34) Cependant, les regroupements généalogiques sont également prégnants dans les romans de Kim Thúy. La tradition vietnamienne confère à la généalogie une importance dont la culture québécoise fait partiellement abstraction : "Quand les Vietnamiens se rencontrent, le village d'origine et l'arbre généalogique sont les deux sujets qui ouvrent la plupart des conversations, parce que nous croyons fermement que nous sommes ce que nos ancêtres ont été, que nos destins répondent aux gestes des vies qui nous ont précédés. » (mãn, p. 53) Comme le rappelle la narratrice de $v i$, la tradition vietnamienne accorde une responsabilité quant à la réussite qui transcende les générations. Elle n'appartient donc pas à une seule personne, mais s'inscrit dans une lignée familiale : «Le succès d'un enfant appartient aux parents et à ses ancêtres. » (vi, p.60) $R u$ défend également le parti pris d'une responsabilité généalogique par le devoir qui unit la narratrice à sa mère dans l'histoire personnelle, mais également l'histoire collective de la diaspora vietnamienne au Québec à la suite du traumatisme de l'exil :

Ma naissance a eu pour mission de remplacer les vies perdues. Ma vie avait le devoir de continuer celle de ma mère.

Je m’appelle Nguyễn An Tịnh et ma mère, Nguyễn An Tĩnh. Mon nom est une simple variation du sien puisque seul un point sous le i me différencie d'elle, me distingue d'elle, me dissocie d'elle. J'étais une extension d'elle, même dans le sens de mon nom. En vietnamien, le sien veut dire "environnement paisible» et le mien, "intérieur paisible». Par ces noms presque interchangeables, ma mère confirmait que j'étais une suite d'elle, que je continuerais son histoire. (ru, p. 11-12) 
La narratrice lie alors ici l'onomastique à la généalogie. Pourtant, la tradition vietnamienne permet également des glissements symboliques dans la généalogie. La narratrice de mãn évoque la multiplicité des figures matriarcales de la tradition vietnamienne. Les tantes, au même titre que les mères, ont une responsabilité quant à l'éducation des enfants: "Souvent, les tantes dans une famille sont appelées "mère" parce qu'elles ont presque le même devoir et le même droit de regard sur le bien-être et l'éducation de l'enfant. » (mãn, p. 109) Ainsi, bien qu'elle connaisse sa mère biologique, la narratrice de mãn est adoptée symboliquement par une amie qui vient combler plusieurs rôles: "Avant même qu'un mot soit prononcé, nous sommes devenues amies et, avec le temps, sœurs. Elle m'a adoptée comme elle a adopté sa fille. » (mãn, p.54) Les regroupements généalogiques se jouent même au cœur du trauma. La cale du bateau lors de la traversée des boat people, du Vietnam au côtes chinoises, devient le théâtre d'un remaniement et, parfois, d'une refonte complète de la généalogie de ses occupants: "Dans cet univers isolé, les amitiés se créaient au moindre lien. Deux camarades de classe devenaient des sœurs, deux natifs d'une même ville s'entraidaient en cousins, deux orphelins formaient une famille.» (vi, p.46) Le rapport à l'altérité de la littérature postexilique de Kim Thúy se matérialise donc à travers une tradition vietnamienne malmenée dans son onomastique et sa généalogie. Au contact de la transculture, le contexte québécois se définit alors comme propre à une hybridation culturelle. 


\section{Hybridations et mémoire culturelles}

Pierre Nepveu, dans L'Écologie du réel, définissait le Québec des écritures migrantes comme « un contexte culturel où l'emprunt est souvent facile, l'hybridation tranquille, le cosmopolitisme inoffensif » (p. 204) et qui était de ce fait favorable à toutes sortes d'hybridation, culturelles, entendons-le, et ainsi littéraires. Il reprenait alors l'exemple de La Québécoite de Régine Robin en 1983, qui, toujours selon lui, montrait une volonté de «noter toutes les différences, faire un inventaire, un catalogue, une nomenclature» (p.204). Cette fragmentation dans la forme de l'expérience de la multiplicité des cultures persiste dans la littérature postexilique de Kim Thúy, dont la forme redondante - le recueil d'anecdotes, qui ne font rarement plus d'une page - dénote une certaine perméabilité. La narratrice de $r u$, qui se fond presque ici avec l'écrivaine, témoigne :

Je raconte des bribes de mon passé comme si elles étaient des historiettes, des numéros d'humoriste ou des contes cocasses de pays lointains aux décors exotiques, aux sons insolites, aux personnages parodiques. (p. 141)

L'écriture est fragmentaire, la chronologie malmenée. Les analepses et les prolepses sont ainsi imprévisibles et suivent la rémanence soudaine des souvenirs. Cette narration décousue fait écho à ce que Pierre Nepveu qualifiait d'expérience du désordre qui caractérise le Québec au présent des écritures migrantes : "L'ici contemporain se définit fondamentalement comme expérience du désordre, d'un désordre encore une fois pas contingent ou momentané, mais persistant, toujours renouvelé.»(p. 207) Régine Robin lui préférait le terme de patchwork culturel pour désigner cette transculture de l'écrit 
migrant, qui méritait, selon elle, la célébration d'une remise en question du nationalisme de souche: «un patchwork de programmes, de cultures, de langues, d'informations et désinformations spécifiques. Quel bonheur! Mélange de tout, bonheur de ce mélange ! (1996, p. 305) L'écriture postexilique de Kim Thúy propose une réponse contrastée à cette exaltation de la transculture. Elle est alors synonyme de désinscription dans l'espace, comme c'est le cas pour le père de la narratrice de vi, qui a « choisi d'être diplomate[...] pour vivre partout dans le monde sans jamais n'appartenir à un lieu » (vi, p. 68). Dans $r u$, la narratrice relate son retour vers le Vietnam, qui fait la synthèse de cette fracture spatiale et culturelle. Dans son restaurant anecdotique, le serveur lui fait remarquer qu'elle n'appartient plus au même groupe que les Vietnamiens restés au pays : «Ce jeune serveur m'a rappelé que je ne pouvais pas tout avoir, que je n'avais plus le droit de me proclamer vietnamienne parce que j'avais perdu leur fragilité, leur incertitude, leurs peurs. Et il avait raison de me reprendre. » (ru, p. 87) Par cette pratique du «dépaysement» et du «repaysement» (Nepveu, 1988, p. 204), la réflexion sur la transculture induit une influence réciproque qui transforme autant l'immigrant que sa société d'accueil, mais qui l'exclut des deux. Le corpus romanesque de Kim Thúy insiste cependant nettement plus sur la suprématie culturelle de l'occident, qui marque bien souvent la période pré-migratoire. La narratrice de $m a \tilde{n}$ évoque son éducation inspirée de la tradition littéraire à travers Bonjour tristesse de Françoise Sagan, Voltaire, Kafka, L'Étranger d'Albert Camus ou encore Les Misérables de Victor Hugo. Dans vi, le père diplomate de la narratrice favorise les cultures extérieures face à l'hybridation en célébrant le contact des cultures américaine et européenne : «Mon père n'a jamais 
aimé Chợ Lớn. Il préférait le centre-ville de Saigon avec ses cafés français et ses bars américains. » (vi, p. 25) De plus, cette coexistence des cultures modifie la perception et la référentialité de certains personnages. La mère de la narratrice compare ainsi son mari à l'acteur américain Clark Gable lorsqu'il s'applique de la brillantine dans les cheveux (vi, p. 19), puisque cette référence à pris la place d'autres références vietnamiennes. Hà, une amie de la famille, évoque également la différence, entre les Vietnamiens et les Américains, de conception de la beauté qui joue en sa faveur :

Je me demandai si ma mère ne l'enviait pas de pouvoir faire preuve de telle désinvolture grâce à la pluie de compliments qu'elle recevait de la part de ses professeurs et collègues américains. Ces derniers célébraient la beauté avec des tablettes de chocolat, des bigoudis, des disques de Louis Armstrong, alors que les Vietnamiens qualifiaient son teint basané de « sauvage ». (vi, p. 34)

Afin de contrebalancer les cultures occidentales dominantes dans la période pré- et post-migratoire, l'écriture de Kim Thúy s'inscrit également dans une perspective de réconciliation et de travail de mémoire qui se lit dans chacun des trois romans. Elle évoque dans mãn le poids de l'histoire pour les Vietnamiens, qui héritent d'un passé collectif. La mère de la narratrice, grâce à son rôle d'actrice de la révolution, a pu empêcher l'exclusion dans les camps de travaux forcés d'une partie de la famille, responsable présumée de la déchéance du pays par son affiliation à l'ancien gouvernement. Elle subit à cet égard le surgissement de l'histoire par les rémanences ponctuelles du passé dans la narration, ici à travers le souvenir des nouveaux occupants de leur grande propriété sous l'ancien régime politique : 
Je me demandais s'il n'était pas insupportable d'avoir son passé planté devant soi. Peut-être espéraient-ils que par compassion les nouveaux occupants ramasseraient, leur redonneraient un coin de la maison, pour que le passé ne soit plus une tare, pour que les gens n'aient plus à noircir au crayon-feutre des visages controversés et des drapeaux de l'ancien régime sur les photos et, surtout, pour réintégrer le passé au présent. (mãn, p. 121-122)

Dans vi, la narratrice évoque même une certaine filiation du poids de l'histoire qui transgresse ainsi les générations: "Parfois, même des parents honnêtes ne résistent pas à la pression du poids de l'histoire d'un peuple qui se transfère d'une génération à l'autre. » (vi, p. 61) L'histoire se transmet alors comme un fardeau culturel, mais cette transmission peut aussi être salvatrice. Dans une perspective cathartique, la narratrice de mãn invite alors sa mère à évacuer le passé par l'écriture en lui offrant de quoi mettre sur le papier ses souvenirs douloureux : "J'avais offert à Maman des cahiers à réglure Seyès comme ceux qu'elle utilisait enfant [...] en espérant qu'elle écrirait notre histoire.» (mãn, p.115) La question se pose alors quant à la possibilité même d'une écriture de l'histoire. Pierre Nepveu indiquait que l'expérience migrante de la mémoire impliquait de «souffrir de ce qui n'existe plus, se souvenir de ce vers quoi on ne peut plus revenir, ou même de ce qui n'a jamais vécu » (1988, p. 200). Cette analyse s'applique au corpus de Kim Thúy, qui mentionne un refus partiel du devoir de mémoire induit par le paramètre temporel et l'effort que demanderait un tel processus : «Les gens sont trop occupés par leur survie quotidienne pour prendre le temps d'écrire leur histoire collective. » ( $r u$, p. 47) Parallèlement, le cheminement historiographique exige des connaissances factuelles qui ne sont pas acquises pour tous les migrants, comme l'explicite vi en l'absence de traces: «Mon 
histoire avait été coupée, réinventée. Aucun objet chez moi ne portait la trace des ancêtres, contrairement à l'autel des ancêtres qui était témoin de tous les mariages, des anniversaires des morts, de la cérémonie du premier jour de l'an depuis au moins cent ans. » (vi, p. 131) L'histoire meurt ainsi parfois par le processus de migration : «Absolument plus personne ne connaîtra la vraie histoire. » (ru, p. 143) Dans son rapport au passé, l'hybridation de la culture migrante vietnamienne infère alors un respect ou, à l'inverse, une transgression de la tradition par le souvenir. Pierre Nepveu expliquait que cette mémoire se caractérise par un « étonnant dilemme: le texte migrant se souvient, croit se souvenir, est hanté par l'originel et l'authentique, mais doit en même temps constater que d'une certaine manière cette hantise est sans objet » (1988, p. 200).

La poétique de l'altérité dans le corpus postexilique de Kim Thúy réinvestit des problématiques latentes en littérature québécoise. Après la saturation des écritures migrantes dans les années 2000, l'auteure à la plume vietnamienne retravaille cette esthétique littéraire en l'inscrivant dans sa propre expérience de l'exil. Son univers est toujours marqué par la prédominance de traces qui ponctuent son écriture, que ce soit à travers ce que Régine Robin nommait l'entre-deux-langues, ici par l'assimilation linguistique de mots vietnamiens, ou le culinaire, qui s'immisce dans le récit, notamment dans mãn. Le rapport à l'altérité se démarque cependant par ses spécificités onomastiques et généalogiques. La littérature au Québec reste ainsi propice aux hybridations, à l'expérience du désordre comme la nommait Pierre Nepveu, notamment par des pratiques identitaires et culturelles qui favorisent la transculture. Le constat de Kim Thúy est néanmoins timoré et ne s'inscrit en aucun cas dans la célébration 
univoque d'un multiculturalisme sans faille. L'exil est aussi parfois perte, oubli, déchirement: «Si tu veux survivre, dépars-toi de ton identité. » (mãn, p. 27)

\section{Bibliographie}

BERROUÊT-ORIOL, Robert. (1986), «L'effet d'exil», Vice Versa, vol. 17, p. 20-21.

BIRON, Michel, François DUMONT et Élisabeth NARDOUT-LAFARGE, (2007) Histoire de la littérature québécoise, Montréal, Boréal.

ChEn, Ying. (1992), La Mémoire de l'eau. Montréal, Leméac.

DESMEules, Christian. (2013), «Littérature québécoise - Les lunettes roses de Kim Thúy », Le Devoir, 30 mars, <http://www.ledevoir.com/culture/livres/374482/leslunettes-roses-de-kim-thuy>.

Dion, Robert. (1997). Le Moment critique de la fiction. Les interprétations de la littérature que proposent les fictions québécoises contemporaines. Québec, Nota bene.

Harel, Simon. (2005), Les Passages obligés de l'écriture migrante. Montréal, Éditions XYZ, coll. « Théorie et Littérature ».

KoKIS, Sergio. (1995), Le Pavillon des miroirs, Montréal, Éditions $\mathrm{XYZ}$, coll. «Romanichels ».

LAFERRIÈRE, Dany. (1985), Comment faire l'amour avec un Nègre sans se fatiguer, Montréal, VLB éditeur.

NEPVEU, Pierre. (1988), L'Écologie du réel. Mort et naissance de la littérature québécoise contemporaine. Montréal, Boréal.

RoBiN, Régine. (1996), «L'impossible Québec pluriel: la fascination de la "souche" », dans Mikhaël Elbaz, Andrée Fortin 
et Guy Laforest (dir.), Les frontières de l'identité : modernité et postmodernisme au Québec, Québec, Presses de l'Université Laval, p. 295-310.

-. (1984), L'Amour du yiddish. Écriture juive et sentiment de la langue (1830-1930), Paris, Éditions du Sorbier.

—. (1983), La Québécoite, Montréal, France/Amérique.

THúY, Kim. (2016), vi, Montréal, Libre Expression.

—. (2013), mãn, Montréal, Libre Expression.

—. (2009), ru, Montréal, Libre Expression.

\title{
Résumé
}

Le renouvellement esthétique suggéré par les écritures migrantes dans les années 1980 a insufflé de nouveaux enjeux en littérature québécoise : flux migratoires, exils, débats sur l'identité et l'altérité au Québec, pour ne citer que les plus prégnants à l'époque. Robert Dion notait que la littérature québécoise s'offrait alors comme une pensée «propice à toutes les hybridations». Même si une certaine saturation de la catégorie évacue ces problématiques à partir des années 2000, Kim Thúy reflète la persistance de ces questionnements dans l'écrit québécois en proposant une nouvelle poétique de l'hybride à travers sa plume d'origine vietnamienne.

\begin{abstract}
The aesthetic renewal suggested by migrant literatures in the $1980 \mathrm{~s}$ breathed life into new issues in Quebecois literature: migratory flows, exiles, debates on identity and alterity in Quebec, not to
\end{abstract}


mention the most significant of the period. Robert Dion noted that Quebecois literature then presented itself as a notion "suitable for all hybridizations". Even if a certain saturation of the category relieves these issues from 2000 on, Vietnamese-Canadian author Kim Thúy reflects the persistence of these examinations in Quebecois writing by proposing a new poetic of the hybrid. 